\title{
Simvastatin bei SPMS gut für die Kognition?
}

Fragestellung: In einer Sekundäranalyse einer randomisierten, kontrollierten Phase-II-Studie (MS-STAT) wurde der Einfluss von hoch dosiertem Simvastatin auf neuropsychologische Testergebnisse und auf die Ergebnisse in Fragebögen zu neuropsychiatrischen Symptomen und zur Lebensqualität bei Patienten mit SPMS untersucht.

Hintergrund: Kognitive Störungen kommen in bis zu $80 \%$ der Patienten mit SPMS vor und beeinträchtigen die Aktivitäten des täglichen Lebens und die Lebensqualität oft stärker als körperliche Behinderungen. Auch neuropsychiatrische Symptome wie Depressivität, Agitiertheit oder Apathie sind häufig und beeinträchtigend. Effektive pharmakologische Behandlungen dieser Symptome existieren nach

Chan D, Binks S, Nicholas JM et al. Effect of high-dose simvastatin on cognitive, neuropsychiatric, and health-related qualityof-life measures in secondary progressive multiple sclerosis: secondary analysis of the MSSTAT randomized, placebocontrolled trial. Lancet Neurol 2017; 16: $591-600$ der aktuellen Datenlage bei SPMS nicht. Sowohl die natürlichen Zusammenhänge und Progressionen von kognitiven und neuropsychiatrischen Beeinträchtigungen sowie Lebensqualität als auch eine mögliche Beeinflussung durch Medikamente sind daher weitgehend unklar.
Patienten und Methodik: Die Autoren analysierten testpsychologische und Fragebogendaten (15 kognitive und 13 neuropsychiatrische Outcomes) von 140 Patienten mit SPMS (70 erhielten 80 mg Simvastatin täglich, 70 Placebo) zur Baseline und nach 24 Monaten.

Ergebnisse: Zur Baseline fanden sich die häufigsten Beeinträchtigungen in einem Screening für Frontalhirnfunktionen (FAB, 60 von 133 Patienten beeinträchtigt), im Arbeitsgedächtnis (PASAT, 62 von 134 Patienten beeinträchtigt) und in der selbstberichteten körperbezogenen Lebensqualität (HRQoL PCS, 68 von 122 Patienten beeinträchtigt). Über 24 Monate zeigte sich die stärkste Verschlechterung in Gedächtnismaßen. Nach 24 Monaten konnte ein kleiner Effekt von Simvastatin für den FAB (1-2 Punkte Verbesserung verglichen mit einem Punkt Verschlechterung in der Placebogruppe) gezeigt werden, ebenso gaben Patienten in der Simvastatin-Gruppe eine höhere körperbezogene Lebensqualität an. Die Verbesserungen in den Exekutivfunktionen waren jedoch nicht mit verminderter Frontalhirnatrophie assoziiert. Für alle anderen Maße zeigte sich kein Effekt von Simvastatin.

Schlussfolgerungen: Hoch dosiertes Simvastatin hat bei SPMS einen kleinen, aber signifikant positiven Effekt auf frontale Exekutivfunktionen sowie auf die körperbezogene Lebensqualität.

\section{- Kommentar von Sven G. Meuth und Andreas Johnen, Münster}

\section{Effekte von Simvastatin auf die Kognition nicht überinterpretieren}

Diese Studie ist die bisher größte und detaillierteste longitudinale Analyse von kognitiven und neuropsychiatrischen Beeinträchtigungen bei Patienten mit SPMS über einen Zeitraum von zwei Jahren. Die Autoren konnten in dieser großen Kohorte zeigen, dass etwa die Hälfte der Patienten ausgeprägte kognitive Störungen (primär Exekutivfunktionen und das Arbeitsgedächtnis betreffend) sowie rund zwei Drittel eine reduzierte (primär körperbezogene) Lebensqualität aufwiesen und auch neuropsychiatrische Symptome in dieser Patientengruppe deutlich von der Norm abwichen. Von insgesamt 28 testpsychologischen Maßen und Fragebogenskalen waren nach 24 Monaten jedoch nur zwei Maße in der Simvastatin- im Vergleich zur Placebogruppe verbessert. Kritisch anzumerken ist hier, dass die Autoren aus inhaltlichen Gründen keinerlei statistische Korrektur für multiple Vergleiche vorgenommen haben. Der Befund einer verbesserten körperlichen Lebensqualität wird hier mit den bereits in der Hauptanalyse gezeigten Verbesserungen der körperlichen Beeinträchtigung (EDSS) unter Simvastatin erklärt. Die gefundene Verbesserung im Test für Frontalhirnfunktionen korrelierte nicht mit dem Ausmaß der Frontalhirnatrophie im strukturellen MRT. Auch zeigten sich in keinen weiteren kognitiven Tests oder den neuropsychiatrischen Fragebögen Gruppenunterschiede nach zwei Jahren, was an der Validität des Ergebnisses zweifeln lässt. Mögliche Veränderungen in Bezug auf Akti- vitäten des täglichen Lebens, die mit den Verbesserungen in den Exekutivfunktionen unter Simvastatin einhergehen könnten, wurden nicht näher untersucht. Insbesondere in Anbetracht der beobachteten deutlichen Progression der Gedächtnisstörung isteinemessbareVerbesserung der Alltagskompetenz fraglich und der zu erwartende positive Effekt auf die Exekutivfunktionen gegen die Nebenwirkungen von hoch dosiertem Simvastatin kritisch abzuwägen. Weitere, hypothesengeleitete Studien mit sensitiveren neuropsychologischen Maßen zur Frontalhirnfunktion verbunden mit ökologischen Maßen zur Messung der Alltagskompetenz sind nötig, um die Nutzen-Risiko-Aspekte einer hoch dosierten Simvastatin-Therapie bei Patienten mit SPMS abschließend einschätzen zu können.

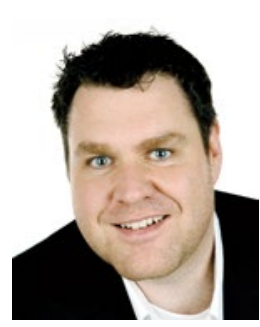

Prof. Dr. med. Dr. rer. nat. Sven Meuth, Münster

Direktor des Instituts für Translationale

Neurologie und stellvertretender Direktor der Klinik für Allgemeine Neurologie, Universitätsklinikum Münster E-Mail: sven.meuth@ukmuenster.de 